\title{
The Re-Emergence of an Old Disease: Chikungunya Fever
}

\author{
Bordi Licia et al.* \\ Laboratory of Virology, National Institute for Infectious Diseases "L. Spallanzani", Rome \\ Italy
}

\section{Introduction}

Until recently, very few physicians in industrialized countries had heard the word "Chikungunya", and fewer knew how to spell it. Chikungunya, a viral infection transmitted by mosquitoes, derives its name from Makonde, a language spoken in south Tanzania, and means "that which bends up", referring to the posture of patients afflicted with severe joint paints characterizing this infection. Chikungunya virus (CHIKV) was first isolated in Tanzania in 1952 (Robinson, 1955) and has come to the world attention recently, when it caused a massive outbreak in the Indian Ocean region and India (Enserik, 2006). Since 1952, CHIKV has caused a number of epidemics, both in Africa and Southeast Asia, many of them having involved hundreds-of-thousands people. In 2005 the largest Chikungunya fever epidemic on record occurred. The most affected region was La Reunion Island, where CHIKV infected more than a third of the population and killed hundred of people. The 2005/2006 outbreak, started from Comoro Islands, rapidly spread to several countries in the Indian Ocean and India (Enserik, 2006; Mavalankar et al., 2007). Compared to earlier outbreaks, this episode was massive, occurred in highly medicalized areas such as La Reunion, and had very significant economic and social impact. More than 1000 imported CHIKV cases have been detected among European and American travellers returning from the affected areas since the beginning of the outbreak in the Indian Ocean region (Fusco et al., 2006; Taubiz et al., 2007), giving rise, in 2007, to the first autochthonous European outbreak in Italy (Charrel \& de Lambellerie, 2008; Rezza et al., 2007). Since 2006, the Regional Office of the French Institute For Public Health Surveillance in the Indian Ocean has conducted epidemiological and biological surveillance for CHIKV infection. During the period December 2006-july 2009, no confirmed case was detected on Reunion Island and Mayotte, but new outbreak were reported in Madacascar. After few years of relative dormancy in Réunion Island, CHIKV transmission has restarted in 2009 and 2010, with one case imported in France (May 2010) (D'Ortenzio et al., 2010). This episode has refreshed the concerns about the possibility of renewed autochthonous transmission in Mediterranean countries.

\footnotetext{
${ }^{*}$ Meschi Silvia ${ }^{1}$, Selleri Marina ${ }^{1}$, Lalle Eleonora ${ }^{1}$, Castilletti Concetta ${ }^{1}$, Carletti Fabrizio ${ }^{2}$, Di Caro Antonino ${ }^{2}$ and Capobianchi Maria Rosaria ${ }^{1}$

${ }^{1}$ Laboratory of Virology, National Institute for Infectious Diseases "L. Spallanzani", Rome, Italy

${ }^{2}$ Laboratory of Microbiology and Infectious Disease Biorepository, National Institute for Infectious Diseases "L. Spallanzani", Rome, Italy
} 


\section{Microbiology}

CHIKV is an alphavirus belonging to the Togaviridae family. Alphaviruses are small and spherical, with a 60-70 nm diameter capsid and a phospholipid envelope. The RNA singlestrand of positive polarity encodes four non structural proteins (nsP1-4) and three structural proteins (C,E1,E2). Viral replication is initiated from the time of attachment of viral envelope to cellular host receptors (Strauss \& Strauss, 1994). Endocitosis of the virus occurs, following which, delivery of the viral nucleocapsid into cytoplasm takes place. The replication cycle is considerably fast, taking around 4hours. Alphaviruses are sensitive to disseccation and to temperatures above $58^{\circ} \mathrm{C}$ (Khan et al., 2002; Strauss J.H. \& StraussE.M., 1994). About 30 species of arthropod-borne viruses are included in the alphavirus genus, antigenically classified into 7 complexes. These viruses are widely distributed throughout the world, with the exception of Antarctica, and 7 of them cause a syndrome similar to Chikungunya fever, arthralgia and rash: Barmah Forest and Ross river viruses (Oceania), O'nyong-nyong and Semliki Forest viruses (Africa), Mayaro (South America), Sindbis and Sindbis-like (Africa, Asia, Scandinavia and Russia) (Taubiz et al., 2007).

\section{Vector and reservoir}

In Asia and the Indian Ocean region the main CHIKV vectors are $A$. aegypti and $A$. albopictus.( Jeandel et al. 2004 ; Zeller, 1998). A larger range of Aedes species (A. furcifer, A. vittatus, A. fulgens, A. luteocephalus, A. dalzieli, A. vigilax, A. camptorhynchites) transmit the virus in Africa, and Culex annulirostris, Mansonia uniformis, and anopheles mosquitoes have also occasionally been incriminated (Jupp P.G. et al., 1981; Jupp P.G. \& McIntosh, 1990; Lam et al., 2001;). In India, the dominant carrier of Chikungunya virus is A. aegypti, which breeds mainly in stored fresh water in urban and semi-urban environments (Yergolkar, 2006). A. albopictus has a wide geographical distribution, is particularly resilient, and can survive in both rural and urban environments. The mosquito's eggs are highly resistant and can remain viable throughout the dry season, giving rise to larvae and adults the following rainy season. Originating from Asia, and initially sylvatic, A. albopictus has shown a remarkable capacity to adapt to human beings and to urbanisation, allowing it to supersede A. aegypti in many places, and to become a secondary but important vector of dengue and other arboviruses (Knudsen, 1995). A. albopictus is zoophilic and anthropophilic, is aggressive, silent, active all-day long, and has a lifespan longer than other mosquitoes (up to 8 weeks) and, in the last decades has expanded to several areas previously known to be Aedes-free (Charrel et al., 2007). It seems that most new introductions of A. albopictus have been caused by vegetative eggs contained in timber and tyres exported from Asia throughout the world. Other emerging events also contributed to the introduction of $A$. albopictus mosquitoes into previously unaffected areas, such as climate change and the increasing use of plastic containers in developing countries. Indeed, climate changes may have several effects on vector biology: increasing temperatures may improve survival at higher latitudes and altitudes, increase the growth rates of vector populations, and alter their seasonality; increased rainfall may have an effect on the larval habitat and population size, and finally an increase in humidity could favourably affect vector survival (Gubler et al., 2001). The use of plastic containers in developing countries, where they are usually not correctly disposed and remain in the environment for years, has also been linked with the spread of the mosquitoes: acting as rain-water receptacles, and being exposed to sunlight, 
they can become perfect "incubators" for mosquito eggs, where the ideal conditions of temperature and humidity are achieved easily and naturally.

Human beings serve as the Chikungunya virus reservoir during epidemic periods. In Africa some animals (monkeys, rodents, and birds) constitute the virus reservoir during not-epidemic periods, sustaining virus circulation in the environment in the absence of human cases. Outbreaks might occur in monkeys when herd immunity is low; the animals develop viraemia but no pronounced physical manifestations (Inoue et al., 2003; Wolfe et al., 2001). An animal reservoir has not been identified in Asia, where humans appear to be the only host.

\section{Clinical manifestation}

\subsection{General features}

After infection with Chikungunya virus, there is a silent incubation period lasting 2-4 days on average (range 1-12 days) (Lam et al., 2001). Clinical onset is abrupt, with high fever, headache, back pain, myalgia, and arthralgia; the latter can be intense, affecting mainly the extremities (ankles, wrists, phalanges) but also the large joints (Hochedez et al., 2006; Lam et al., 2001; Quatresous, 2006; Robinson, 1955; Saxena et al., 2006). Skin involvement present in about $40-50 \%$ of cases, and consists of (1) a pruriginous maculopapular rash predominating on the thorax, (2) facial oedema, or (3) in children, a bullous rash with pronounced sloughing, and (4) localised petechiae and gingivorrhagia (mainly in children) (Brighton et al., 1983; Fourie \& Morrison, 1979). Radiological findings are normal, and biological markers of inflammation (erythrocyte sedimentation rate and C-reactive protein) are normal or moderately elevated (Fourie \& Morrison, 1979; Kennedy et al., 1980). Iridocyclitis and retinitis are the most common ocular manifestations associated with Chikungunya fever; less frequent ocular lesions include episcleritis. All ocular manifestations have a benign course with complete resolution and preservation of vision. Retinitis shows gradual resolution over a period of 6 to 8 weeks (Mahendradas et al., 2008). CHIKV infection seems to elicit long-lasting protective immunity, and experiments performed using animal models have shown a partial cross-protection among CHIKV and other alphaviruses (Edelman et al., 2000; Hearn Jr. \& Rainey, 1963).

\subsection{Arthralgia}

Erratic, relapsing, and incapacitating arthralgia is the hallmark of Chikungunya, although it rarely affects children. These manifestations are normally migratory and involve small joints of hands, wrists, ankles, and feet with pain on movement. Symptoms generally resolve within 7-10 days, except for joint stiffness and pain: up to $12 \%$ of patients still have chronic arthralgia three years after onset of the illness. Arthralgia experienced by CHIKF patients closely resembles the symptoms induced by other viruses like Ross River Virus (RRV) and Barmah Forest virus (BFV) (Jacups et al, 2008; Mahalingam et al., 2002). Such alphavirusinduced arthralgia mirrors rheumatoid arthritis, a condition which is characterised by severe joint pains due to inflammation and tissue destruction caused by inflammatory cytokines such as IL-1b, IL-6 and TNF-a (Barksby et al., 2007). It is thus plausible that CHIKV infection induces similar pro-inflammatory cytokines that cause arthralgia, explaining why joint pains are constant ailments of many patients infected with CHIKV even years after recovery from the initial febrile phase (Lakshmi et al., 2008). More recently, global analyses on the specific involvement of cytokines and chemokines have showed that IL-1b, IL-6, and RANTES were associated with disease severity ( $\mathrm{Ng}$ et al., 
2009). Moreover, since high concentrations of these pro-inflammatory factors were found in the joints of humans afflicted with RRV-induced polyarthritis, they probably have a causative role in chronic joint and muscle pains that plague patients (Lidbury et al., 2008). The finding that aberrant Type I interferon signalling in mice led to severe forms of CHIKF (Couderc et al., 2008) further highlighted the important role cytokines play in the pathology of CHIKV infection.

\subsection{Other pathologies}

Chikungunya is not generally considered to be a life-threatening disease. Usually the clinical course is fairly mild, but fatal cases directly or indirectly linked to infection with CHIKV have been observed during the Indian-Ocean outbreak (Josseran et al., 2006). The main evidence of a mortality linked to Chikungunya fever epidemics has been obtained in La Reunion, Mauritius, and India by comparing expected and observed mortality data. In all cases, during the months when the epidemics were raging, the observed mortality significantly exceeded the expected one. In particular, in La Reunion the monthly crude death rates in February and March 2006 were 34.4\%and 25.2\% higher, respectively, than expected. These corresponded to 260 excess deaths (an increase of 18.4\%) with a rough estimate of the case-fatality rate for Chikungunya fever of $\approx 1 / 1,000$ cases. The case-fatality rate calculated on increased crude death rates in Mauritius and Ahmedabad, India, is substantially higher than that calculated in La Reunion: approximately $4.5 \%(15,760$ confirmed or suspected cases and 743 excess deaths) and 4,9\% (60,777confirmed or suspected cases and 2,944 excess deaths), respectively (Beesoon et al., 2008; Mavalankar et al., 2008). These differences may be attributed to many factors (greater disease severity, preexisting patient conditions, different patient management, or coincident excess deaths from other causes) but may also be due to a different efficacy of the surveillance systems for Chikungunya fever, that probably worked poorly in Mauritius and India, leading to underestimating the total number of cases (Fusco et al., 2010). The possible link between CHIKV infection and multiorgan failure is still under investigation.

Neurological complications such as meningo-encephalitis were reported in a few patients during the first Indian outbreak in 1973, and during the 2006 Indian outbreak (Chatterjee et al., 1965; Ravi, 2006). The possible mechanisms underlying these processes remain unknown. Studies performed on animal models showed that CHIKV-infected young mice had weakness and walking difficulties which could be due to necrosis and inflammation of skeletal muscles (Ziegler et al., 2008). CHIKV antigens and viral replication have been detected in human myogenic precursors such as satellite cells but not in muscle fibers (Ozden et al., 2007), suggesting that muscle satellite cells could be potential virus reservoirs. The pathologic symptoms of encephalitis owing to CHIKV infection as well as central nervous system (CNS) infections (Chatterjee \& Sarkar, 1965) were expected, since in vitro experiments showed that the virus could infect and replicate for extended periods in mouse brain cells (Precious et al., 1974). More recently, it was found that mouse CNS tissues such as the choroid plexi could also be targets of CHIKV, lending more credence to the fact that CHIKV infections do affect CNS cells and tissues (Couderc et al., 2008). Work is currently underway by several research groups around the world to decipher this mechanism in CHIKV infections. Moreover, during the 2006 Indian-Ocean outbreak, rare cases of GuillainBarré syndrome (GBS) associated with CHIKV infection have been described (Lebrun et al., 2009; Wielanek et al., 2007). 
Other rare complications described after CHIKV infection are mild hemorrhage, myocarditis, hepatitis (Lemant et al., 2008) .

\section{Diagnosis}

Diagnosis of infection with CHIKV is based on molecular biology (RT-PCR) and serology methods. The first one is useful during the initial viraemic phase, at the onset of symptoms and normally for the following 5-10 days, when CHIKV RNA reaches very high levels (viral loads of $3.3 \times 10^{9}$ copies $/ \mathrm{ml}$ ) and can be detected (Carletti et al., 2007; Parola et al., 2006). Afterwards, the diagnosis is based on serological methods (ELISA, immunofluorescence, hemoagglutination inhibition $(\mathrm{HI})$ and infectivity neutralization $(\mathrm{Nt})$ ).

IgM specific against CHIKV are detectable 2-3 days after the onset of symptoms by ELISA immunofluorescent assay and persists for several weeks, up to 3 months (Litzba et al, 2008; Sam \& AbuBakar, 2006); rarely, IgM can be detected for longer periods, up to 1 year. IgG specific against CHIKV appear soon after IgM antibodies (2-3 days) and persists for years. Testing of a couple of sera collected in the acute and the convalescent phases of the disease is mandatory for the identification of recent infection using serology methods that cannot distinguish $\operatorname{IgG~} \mathrm{Ab}$ from $\operatorname{IgM~} \mathrm{Ab}$ (i.e. $\mathrm{HI}$ and $\mathrm{Nt}$ ). It is also very useful to confirm results obtained with other methods, especially taking into account the although rare persistence of IgM antibodies. Viral isolation can be performed from serum of infected patient on insect or mammalian cell lines (i.e. C6/36 or Vero E6) during the early phase of the disease, when the viral load is very high and the immune response is still not detectable; however it is useful only for epidemiology or pathogenesis studies or for thorough molecular characterization (Fusco et al., 2010). The sensitivity and specificity of rapid bedside tests commercially available are poorly established, and the possibility of false-positive reactions resulting from cross-reactivity with dengue or other arboviruses such as o'nyong-nyong virus has to be considered (Blackburn et al., 1995). Serologically, chikungunya virus is most closely related to o'nyong-nyong virus and is a member of the Semliki Forest antigenic complex. Individual serological testing is not particularly useful, except when faced with atypical or severe forms, or in travellers returning from an epidemic zone (Pile et al., 1999).

\section{Treatment}

Currently, there are no available specific therapeutics against CHIKV. Treatment is purely symptomatic and can include rest, fluids, and medicines to relieve symptoms of fever and aching, such as ibuprofen, naproxen, acetaminophen, or paracetamol. Non-steroidal antiinflammatory drugs (NSAIDs) are primarily used to treat inflammation but high doses, administrated to control arthralgia, could cause thrombocytopenia, gastrointestinal bleeding, nausea, vomiting and gastritis (Jain et al, 2008; Pialoux et al., 2007). Steroids have been occasionally used but their efficacy was not significant (Taubitz et al., 2007). Some time ago chloroquine, a drug useful for prophylaxis and treatment of malaria, showed promising results for treating chronic Chikungunya arthritis (Brighton, 1984), while a recent trial conducted on French Reunion Island proved that there is currently no justification for the use of chloroquine to treat acute chikungunya diseases (De Lamballerie et al., 2008). However, the usefulness of chloroquine in the treatment of Chikungunya infection deserves further investigation that could take advantage on the availability of a non-human primate animal model (Labadie et al., 2010). Ribavirin (200 mg twice a day for seven days) given to 
patients who continued to have crippling lower limb pains and arthritis for at least two weeks after a febrile episode, had a direct antiviral property against $\mathrm{CHIKV}$, leading to faster resolution of joint and soft tissue manifestations (Ravichandran \& Manian, 2008). Briolant and collegues screened various active antiviral compounds against viruses of the Alphavirus genus in vitro and demonstrated that 6-azauridinet was more effective against $\mathrm{CHIKV}$, as compared to ribavirin. Moreover, the combination of IFN-alpha2b and ribavirin had synergistic antiviral effect on Chikungunya virus (Briolant et al., 2004).

It is widely recognized that passive vaccination is an appropriate preventive and therapeutic option for many viral infections in human, including those spread by viral vertical transmission, especially when no alternative therapy is available (Dessain et al., 2008). Human polyvalent immunoglobulins purified from plasma samples obtained from donors in the convalescent phase of CHIKV infection exhibited a high in vitro neutralizing activity and a powerful prophylactic and therapeutic efficacy against CHIKV infection in vivo in mouse models (Couderc et al., 2009). Due to the demonstrated efficacy of human antiCHIKV antibodies in a mouse model, purified polyvalent CHIKIg (commercialized under the brand Tégéline) could be used in humans for prevention and treatment, especially in individuals at risk of severe CHIKV disease, such as neonates born to viraemic mothers and adults with underlying conditions. Polyclonal immune globulins present the advantage of a broad reactivity but the therapeutic intervention is limited, due to the short viremia in acute phase of CHIKV infection: thus the only benefit this treatment has to offer would be to help reducing viremia faster (Kam et al., 2009). As an alternative, more specific human monoclonal antibodies (MAbs) could be used. In a recent study two unique human mAbs, specific for the CHIKV envelope glicoproteins, strongly and specifically neutralized CHIKV infection in vitro (Warter et al., 2011).

\section{Prevention}

Although no licensed vaccines are currently available for CHIKV, potential vaccine candidates have been tested in humans and animals with varying success. Due to the easiness in preparation, the first developed vaccines were formulations of whole-virus grew on cells and inactivated either by formalin or tween-ether (Eckels et al., 1970; Harrison et al., 1967, 1971; White et al., 1972).

Further vaccines are focused on attenuated strains of CHIK obtained after serial passages in cells cultures (Edelman et al., 2000; Levitt et al., 1986). One of these promising candidates is TSI-GSD-218, a serially passaged and plaque-purified live CHIK vaccine, tested for safety and immunogenicity in human Phase II trials by the US Army Medical Research Institute (Edelman et al., 2000). Seroconversion was obtained in $98 \%$ of vaccinees volunteers by day 28 and neutralizing antibodies persisted in $85 \%$ of cases at one year after immunization. However transient arthralgia occurred in $8 \%$ of the volunteers. Some chimeric candidates vaccines were developed using either Venezuelan equine encephalitis (VEEV) attenuated vaccine strain TC-83, a naturally attenuated strain of eastern equine encephalitis virus (EEEV), or Sindbis virus (SV) as a backbone and the structural protein genes of CHIKV. Vaccinated mice were fully protected against disease and viraemia after CHIKV challenge (Wang et al., 2008). The maturity of reverse genetic technology has provided unprecedented opportunities for manipulation of the alphaviral genome to improve attenuation strategies. Thus, unlike traditional attenuation approaches that rely on cell culture passages, which typically result in attenuation that depends only on small numbers of attenuating point 
mutations, alternative genetic strategies such as viral chimeras offer the promise of more stable attenuation (Kennedy et al., 2011). In addition to the risk of reactogenicity, attenuation based on small numbers of mutations can also result in residual alphavirus infectivity for mosquito vectors. This risk, which was underscored by the isolation of the TC-83 VEEV vaccine strain from mosquitoes in Louisiana during an equine vaccination campaign designed to control the 1971 epidemic (Pedersen et al., 1972), is especially high when a vaccine that relies on a small number of point mutations is used in a nonendemic location that could support a local transmission cycle. In a recent study chimeric alphaviruses, encoded CHIKV-specific structural genes (but no structural or nonstructural proteins capable of interfering with development of cellular antiviral response) induced protective immune response against subsequent CHIKV challenge (Wang et al., 2011). More in detail, recombinant chikungunya virus vaccine, comprising a non-replicating complex adenovirus vector encoding the structural polyprotein cassette of chikungunya virus, consistently induced in mice high titres of anti-chikungunya virus antibodies that neutralised both an old Asian isolate and a Réunion Island isolate from the recent epidemic (Wang et al., 2011).

A novel CHIK vaccine candidate, CHIKV/IRES, was generated by manipulation of the structural protein expression of a wt-CHIKV strain via the EMCV IRES (Plante et al., 2011). In particular, the internal ribosome entry site (IRES) from encephalomyocarditis virus replaced the subgenomic promoter in a cDNA CHIKV clone, thus altering the levels and host-specific mechanism of structural protein gene expression. This vaccine candidate exhibited a high degree of murine attenuation that was not dependent on an intact interferon type I response, highly attenuated and efficacious after a single dose.

Another approach was the selective expression of CHIK viral structural proteins recently obtained by Akata and collegues using virus-like particles (VLPs) in vitro, that resemble replication-competent alphaviruses (Akahata et al., 2010). Immunization of monkeys with these VLPs elicited neutralizing antibodies against envelope proteins from different CHIKV strains and obtained antibodies transferred into mice protective against subsequent lethal CHIKV challenge. The last frontier in the approach of CHIK vaccine design is the DNA vaccine strategy. An adaptive constant-current electroporation technique was used to immunize mice (Muthumani et al., 2008) and rhesus macaques (Mallilankaraman et al., 2011) with an intramuscular injection of plasmid coding for the CHIK-Capsid, E1 and E2. Vaccination induced robust antigen-specific cellular and humoral immune responses in either case.

To date a number of CHIKV vaccines have been developed, but none have been licensed. While a number of significant questions remain to be addressed related to vaccine validation, such as the most appropriate animal models (species, age, immune status), the dose and route of immunization, the potential interference from multiple vaccinations against different viruses, and last, the practical cost of the vaccine, since most of the epidemic geographical regions belong to the developing countries, there is real hope that a vaccine to prevent this disease will not be too long in arriving.

Since a vaccine is not available actually, protection against mosquito bites and vector control are the main preventive measures. Individual protection relies on the use of mosquito repellents and measures in order to limit skin exposure to mosquitoes. Bednets should be used during the night in hospitals and day-care facilities but Aedes mosquitoes are active allday-long. Control of both adult and larval mosquito populations uses the same model as for dengue and has been relatively effective in many countries and settings. Breeding sites must be removed, destroyed, frequently emptied, and cleaned or treated with insecticides. Large- 
scale prevention campaigns using DDT have been effective against $A$. aegypti but not $A$. albopictus. Control of $A$. aegypti has rarely been achieved and never sustained (Reiter et al., 2006). Recent data show the different degrees of insecticide resistance in $A$. albopictus and $A$. aegypti (Cui et al., 2006). However, vector control is an endless, costly, and labour-intensive measure and is not always well accepted by local populations, whose cooperation is crucial. Control of CHIKV infection, other than use of drugs for treatment of disease, development of vaccines, individual protection from mosquitoes and vector control programs, also involves surveillance that is fundamental for early identification of cases and quarantine measurement. A model used in investigation of the transmission potential of CHIKV in Italy has proven useful to provide insight into the possible impact of future outbreaks in temperate climate regions and the effectiveness of the interventions performed during the outbreak (Poletti et al., 2011).

\section{Geographic distribution and map}

Chikungunya fever has an epidemiological pattern with both sporadic cases and epidemics in west Africa, from Senegal to Cameroun, and in many other African countries (Democratic Republic of Congo, Nigeria, Angola, Uganda, Guinea, Malawi, Central African Republic, Burundi, and South Africa). Moreover, many epidemics occurred in Asia (Burma, Thailand, Cambodia, Vietnam, India, Sri Lanka, Timor, Indonesia, and the Philippines) in the 1960s and in the 1990s (Jain et al., 2008; Pialoux et al., 2007).

Major epidemics appear and disappear cyclically, usually with an inter-epidemic period ranging from 7 to 20 years. The huge outbreak that increased concern about CHIKV started in Kenya in 2004, where the seroprevalence rates reached 75\% in Lamu island (Pialoux et al., 2007), before reaching the Comores, Seychelles, and Mauritius islands. The virus reached La Reunion island, a French overseas district, in March-April 2005, probably as a result of importation of cases among immigrants from the Comores. The outbreak had two phases: after some thousands of cases which occurred in March-April 2005, very few cases were reported during the austral winter, while the second epidemic peak arose in the initial months of 2006. For the first time, a substantial number of deaths (254) were attributed, directly or indirectly, to CHIKV. From late 2005 onwards, hospitals in some Indian states found themselves swamped with patients complaining of fever and joint pain, which turned out to be Chikungunya fever (Fusco et al., 2010). The World Health Organization Regional Office for South-East Asia has reported that 151 districts in nine states/provinces of India have been affected by Chikungunya fever between February and October 2006 (Pialoux et al., 2007).

Several imported cases were reported in industrialized countries among travellers returning from endemic areas, mainly tourists and immigrants (Depoortere \& Coulombier, 2006). In particular, many cases were detected in early 2006, when the outbreak involved the Indian Ocean islands. The Indian Ocean islands, India, and Malaysia are popular tourist destinations. According to the World Tourism Organization, an estimated 1474218 people travelled from Madagascar, Mauritius, Mayotte, Reunion, and the Seychelles to European countries in 2004 (Depoortere \& Coulombier, 2006; Parola et al., 2006).

The European country with the highest number of imported cases was France, especially the south-eastern region of Provence-Alpes-Côte d'Azur, and Marseille in particular, home to a large Comorian community (Cordel et al., 2006; Hochedez et al., 2007). Other European countries that reported imported cases include Belgium, Bosnia, Czech Republic, Croatia, 
Germany, Greece, Italy, Serbia, Spain, Switzerland, Norway, and the United Kingdom (Beltrame, A. 2007; Deporteere \& Coulombier, 2006; Fusco, F.M. 2006; Pialoux et al., 2007; Taubitz et al., 2007). In 2006, CHIK fever cases have also been reported in traveller returning from known outbreak areas to Canada, the Caribbean (Martinique), and South America (French Guyana). During 2005-2006, 12 cases of CHIK fever were diagnosed serologically and virologically at CDC in travellers who arrived in the United States from areas known to be epidemic or endemic for CHIK fever, and 26 additional imported cases with onset in 2006 underscores the importance of recognizing such cases among travellers (CDC, 2006; CDC 2007).

Moreover, CHIKV gave rise in 2007 to the first autochthonous European outbreak in Italy, in the northern region of Emilia-Romagna (Rezza et al., 2007; Charrel et al., 2008).

In June 2007, an Indian citizen returned to Italy after a visit to relatives in Kerala, India, developed 2 episodes of fever. During the second febrile episode, he visited his cousin in Castiglione di Cervia. The cousin had an onset of symptoms, with fever and arthralgia, on July 4 . This sequence of events started the first Chikungunya fever outbreak in a temperate country, that lasted approximately 2 months with a total 247 cases of Chikungunya fever occurred in the region (217 laboratory-confirmed, 30 suspected) (Fusco et al., 2010). A unique sequence of events seems to have contributed to the establishment of local transmission in Emilia-Romagna: the high concentration of competent vectors A. albopictus in the area at the time of arrival of the index case, the presence of a sufficient human population density and the temporal overlapping of arthropod activity (seasonal syncronicity) (Charrel et al., 2008; Rezza et al., 2007).

During 2008, cases of Chikungunya fever have been reported from many countries in Asia other than India, as well as active epidemics from Singapore, Sri Lanka, and Malaysia (Leo et al., 2009).

Since 2006, the Regional Office of the French Institute For Public Health Surveillance in the Indian Ocean has conducted epidemiological and biological surveillance for CHIKV infection. During the period December 2006-July 2009, no confirmed case was detected on Reunion Island and Mayotte, but new outbreaks were reported in Madacascar. After few years of relative dormancy in Réunion Island, in August 2009, a cluster of cases was identified on the western coast of Réunion Island (D'Ortenzio et al., 2009) and, subsequently, an outbreak of CHIKV infection was described on Réunion Island in 2010 (D'Ortenzio et al., 2011). Moreover, recent publications described cases of Chikungunya fever in tourist returning from Maldives, confirming the circulation of the virus by the end of 2009 (Pfeffer et al., 2010; Receveur et al., 2010)

These episodes have refreshed the concerns about the possibility of renewed autochthonous transmission in Mediterranean countries and highlight the need for surveillance in countries where emerging infections may be introduced by returning travellers. Travellers can serve as sentinel population providing information regarding the emergence or re-emergence of an infectious pathogen in a source region. Travellers can thus act as carriers who inadvertently ferry pathogens that can be used to map the location, dynamics and movement of pathogenic strains (Pistone et al, 2009). Thus, with the increase in intercontinental travel, travellers can provide insights into the level of the risk of transmission of infections in other geographical regions.

The geographic range of CHIKV is mainly in Africa and Asia (Fig. 1) 


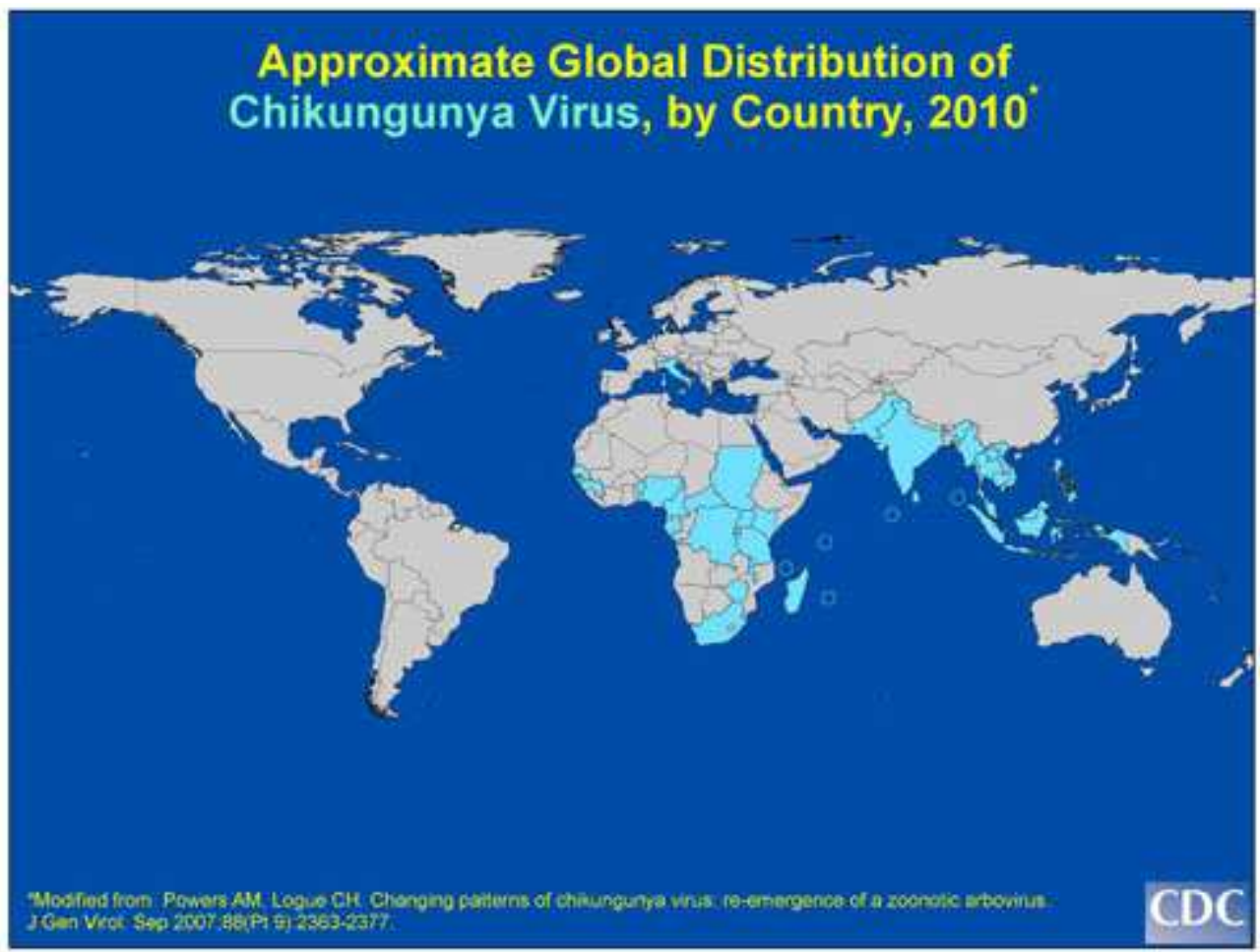

Fig. 1. Geographical distribution of CHIKV shown in the most recent map coming from the CDC's Traveler's Health website (http://wwwn.cdc.gov/travel/default.aspx).

\section{Phylogenesis}

Three lineages of CHIKV, with distinct genotypic and antigenic characteristics, have been identified. Isolates that caused the 2004-06 Indian Ocean outbreak form a distinct cluster within the large eastern and central Africa phylogenetic group, in addition to the Asian and west African phylogenetic groups (Powers et al., 2000; Schuffenecker et al., 2006). Phylogenetic analysis of CHIKV strains circulating in A. Albopicus-humans transmission cycles, obtained during outbreaks, have identified the independent acquisition of a common mutation in E1 glycoprotein (E1gp), namely A226V, in strains isolated from different geographic regions (Schuffenecker et al., 2006; de Lambellerie et al., 2008). This mutation, together with M269V, D284E mutations of E1 CHIKV glycoprotein have been described as molecular signatures of the Indian Ocean outbreak (Arankalle et al., 2007; Tsetsarkin et al., 2007; Vazeille at al., 2007). In particular, the A226V mutation, which was absent in the strains isolated during the initial phases of the outbreak in Réunion, appeared in $>90 \%$ of the isolates after Dicember 2005. This change could be related to virus adaptation to the mosquito vector species. Together with the lack of herd immunity, this might explain the abrupt and escalating nature of the Reunion outbreak. Has been clearly demonstrated that the A226V mutation is able to increase viral fitness in the Aedes albopictus vector (Tsetsarkin 
et al., 2007; Vazeille et al., 2007), that, in turn, may expand the potential for CHIKV to diffuse to the Americas and Europe, due to the widespread distribution of this vector, in particular in Italy (Knudsen, 1995). In a previous paper we characterized 7 viral isolates (5 imported and 2 autochthonous cases), with respect to the molecular signatures of the Indian Ocean Outbreak in E1, particularly the A226V mutation. Imported cases included 3 returning from Mauritius in 2006 and 2 returning from India in 2006 and 2007, respectively; the autochthonous cases occurred during the 2007 Italian outbreak (Bordi et al., 2008). CHIKV sequences of a $1013 \mathrm{bp}$ fragment of E1 gene (nucleotide positions 10145-11158, respect to the reference strain S27) have been analyzed (Fig.2).

All 7 isolates carried the M269V and D284E Indian Ocean signatures while the A226V mutation was present in all the isolates imported from Mauritius, in the autochthonous cases from the Italian outbreak and in the isolate imported from India in 2007, but was absent in the case imported from India in 2006.

Our findings indicated that, during 2006 and 2007, multiple strains have been imported to Italy from countries where explosive Chikungunya outbreaks were ongoing. All the strains isolated in Italy, both imported and autochthonous, displayed two molecular signatures of

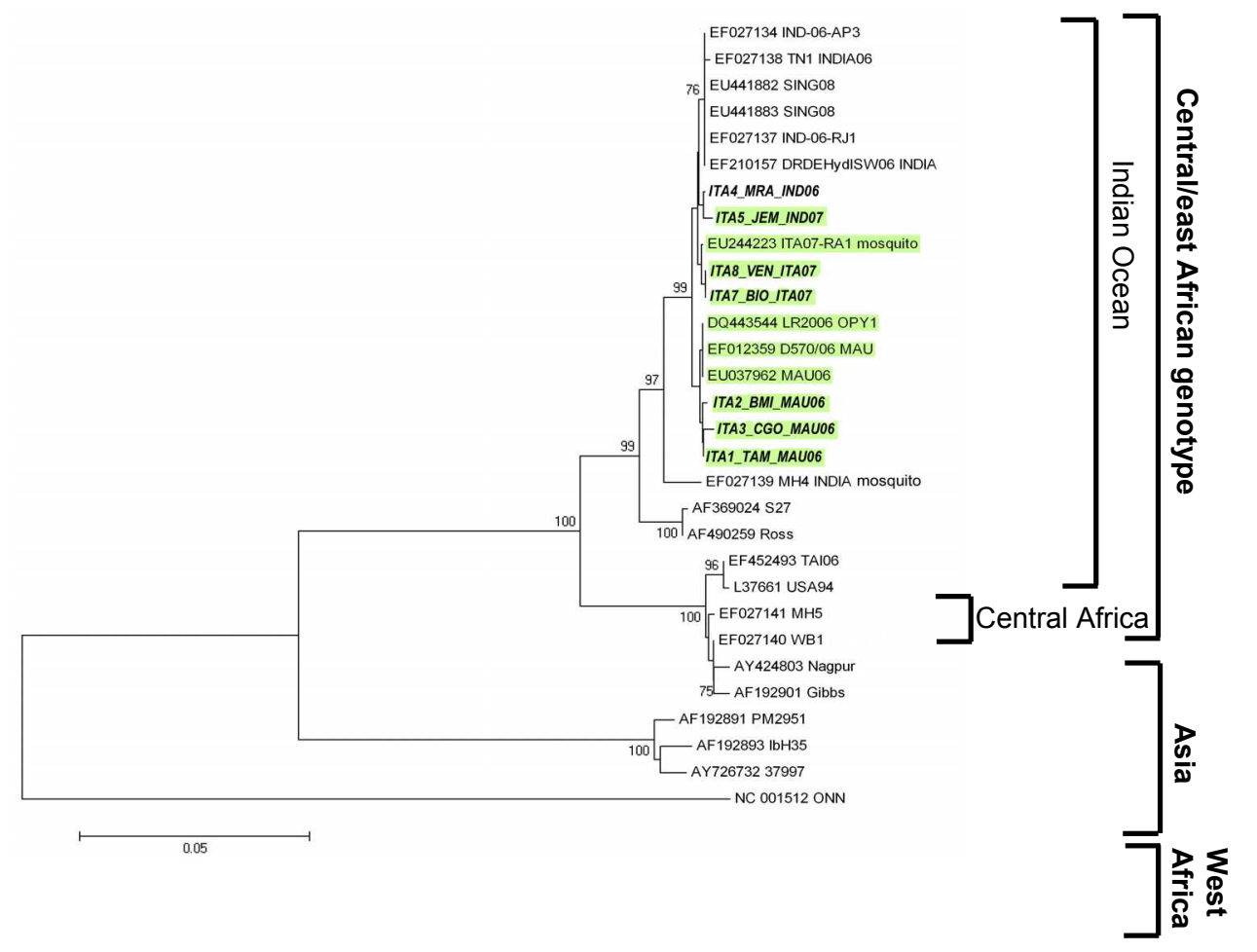

Fig. 2. Phylogenetic tree of CHIKV strains performed on partial E1 gene

CHIKV sequences of a 1013 bp fragment of E1 gene (nucleotide positions 10145-11158, respect to the reference strain S27) have been analyzed. The strains isolated from human cases in Italy are in bold (Bordi et al., Clin Infect Dis, 2008) 
the Indian Ocean outbreak (M269V and D284E). Concerning the A226V mutation, this was present in all imported and autochthonous cases, with the exception of the isolate imported from the Indian subcontinent in 2006. The absence of this mutation in the isolate imported in 2006 from India was in agreement with published data (Arankalle et al., 2007), and with available GenBank sequence data, indicating that the virus strains circulating in India in 2006 lacked this mutation.

The presence of $\mathrm{A} 226 \mathrm{~V}$ in the isolate imported from India in July 2007 and in the isolates from the 2007 Italian outbreak (originating from a case imported from India) supports the view that the virus envelope sequence of strains from India changed over time, acquiring after 2006 the E1 mutation associated with enhanced fitness in Aedes albopictus. So it appears that the acquisition and fixation of the A226V mutation may be a common pathway of chikungunya explosion in epidemic areas, in a parallel interplay with the mosquito vector dynamics. Noteworthy, the outbreak in Singapore, where the A226V mutation was absent, has been rapidly controlled.

\section{Immune-pathogenesis}

Given the expanding geographic range of CHIKV and its potential to rapidly cause large scale epidemics, it has become important to understand the immune and pathogenic mechanisms active during CHIKV infections in order to guide the development of targeted and effective control and treatment strategies.

In a review the possible interactions of the immune system with the different stages of the CHIKV life cycle have been discussed (Kam et al., 2009). The first encounter of CHIKV with human host is intradermal inoculation by the mosquito: replication of the virus starts at the site of inoculation. Different resident cell types are present in this location, including keratinocytes, dermal dendritic cells (DCs), Langerhans cells (LCs), and dermal macrophages, cells involved in the innate immune response.

The innate immune response is the first barrier against viruses, being able to inhibit viral replication through cytolytic and non-cytolytic mechanisms. IFN system plays an important role in limiting virus spread at an early stage of infection. In vitro growth of all tested alphaviruses can be greatly suppressed by the antiviral effects of Interferon- $\alpha / \beta$ (IFN$\alpha / \beta$ ) when it is added to cells prior to infection, and, more specifically, CHIKV replication is significantly influenced by type I and II IFNs (Courderc et al., 2008; Schilte et al., 2010; Sourisseau et al., 2007). The finding that aberrant Type I interferon signalling in mice led to severe forms of CHIKF (Couderc et al., 2008) further highlighted important roles cytokines play in the pathology of CHIKV infection. Moreover, in a very recent study Wauquier and colleague demonstrated that CHIKV infection in humans elicit strong innate immunity involving the production of numerous proinflammatory mediators. Interestingly, high levels of Interferon IFN- $a$ were consistently found. Production of interleukin (IL) 4, IL-10, and IFN- $\gamma$ suggested the engagement of the adaptive immunity. This was confirmed by flow cytometry of circulating $\mathrm{T}$ lymphocytes that showed a CD8+ T lymphocyte response in the early stages of the disease, and a CD4+ T lymphocyte mediated response in the later stages (Wauquier et al., 2011).

It was already known that skin cell fibroblasts were susceptible to CHIKV infection (Sourisseau et al.,2007); recently has also been demonstrated that CHIKV antigens could be detected in vivo in the monocytes of acutely infected patients (Her et al, 2010). CHIKV 
interactions with monocytes, and with other blood leukocytes, induced a robust and rapid innate immune response with the production of specific chemokines and cytokines. In particular, high levels of IFN- $\alpha$ were rapidly produced after CHIKV incubation with monocytes. The identification of monocytes during the early phase of CHIKV infection in vivo is significant as infected monocyte/macrophage cells have been detected in the synovial tissues of chronically CHIKV-infected patients, and these cells may behave as the vehicles for virus dissemination. This may explain the persistence of joint symptoms despite the short duration of viraemia (Her et al., 2010).

Since the A226V mutation has been associated with enhanced replication and fitness of CHIKV in A. albopictus vector and has also been shown to modulate cholesterol requirement for infection of insect cells (Tsetsarkin et al., 2007), in a recent paper we investigated the possible involvement of A226V mutation in enhancing human pathogenesis in non vector hosts, by testing the replication competence in primate cell cultures of two isolates, differing for the presence or absence of this mutation (Bordi et al., 2011). We observed that the presence of $\mathrm{A} 226 \mathrm{~V}$ mutation did not influence the replication kinetics on primate cells. Moreover, the time course of appearance of cytopathic effect (CPE) and of cells immunostained with CHIKV-specific antiserum, was very similar for both the isolates, as well as the shape of the virus-positive multicellular foci, thus suggesting a similar mechanism of spread of the virus in the infected cell cultures.

In addition, we considered the possibility that the A226V mutation could be associated with partial resistance to the inhibitory action of IFN- $\alpha$ in classical experiments of inhibition of virus replication. Surprisingly, the A226V-carrying strain was more susceptible to the antiviral action of recombinant IFN- $\alpha$. (Fig.3)

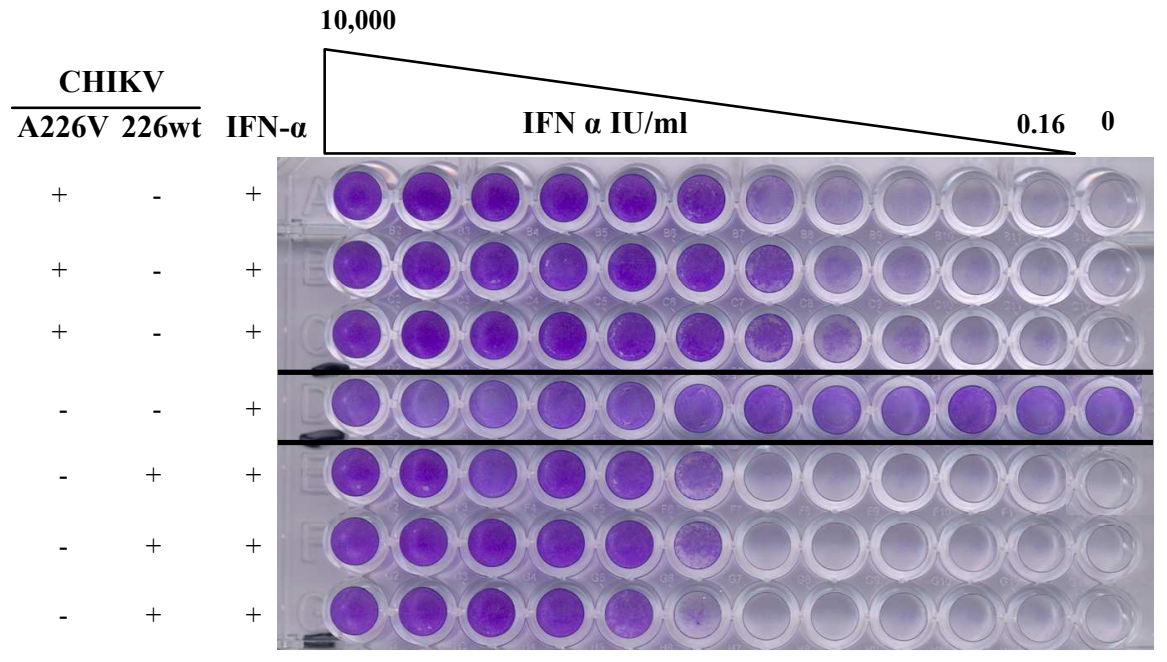

Fig. 3. Dose-dependent reduction of viral CPE by recombinant IFN- $\alpha$.

In vitro experiments of inhibition of virus replication by recombinant IFN- $\alpha$ on Vero E6 cells showing a dose-dependent reduction of $\mathrm{CPE}$ for both isolates: the A226V, carrying isolate and the wt (Bordi et al., New Microbiol, 2011). 
Overall, our result did not support the concept that A226V mutation confers a replicative advantage in primate cell cultures, neither supported the possibility that partial resistance to the inhibitory action of IFN- $\alpha$ could account for the explosive spread of the mutated strain in the human population in the countries where this mutation had occurred. However, the possibility that the interplay between the virus and the innate defence system may act at different levels of the virus/host interaction is to be taken into consideration, by exploring, for instance, other steps of the IFN response activation.

At the moment, understanding CHIKV immuno-biology is still in its infancy and there is a long way to go before answers related to the interaction between virus and host immunity will be obtained. These will certainly be important in designing novel antiviral control strategies against the spread of CHIKV infection.

\section{References}

Akahata W., Yang Z.Y., Andersen H., et al. A VLP vaccine for epidemic Chikungunya virus protects nonhuman primates against infection. Nat Med, 2010; 16: 334-338

Arankalle V.A., Shrivastava S., Cherian S., et al. Genetic divergence of Chikungunya viruses in India (1963-2006) with special reference to the 2005-2006 explosive epidemic. J Gen Virol, 2007; 88: 1967-1976

Barksby H.E., Lea S.R, Preshaw P.M., et al. The expanding family of interleukin-1 cytokines and their role in destructive inflammatory disorders. Clin Exp Immunol, 2007; 149: 217-225

Beesoon S., Funkhouser E., Kotea N., Spielman A., et al. Chikungunya fever, Mauritius, 2006. Emerg Infect Dis, 2008; 14: 337-338

Beltrame A., Angheben A., Bisoffi Z., et al. Imported Chikungunya infection, Italy. Emerg Infect Dis, 2007; 13: 1264-1266

Blackburn N.K., Besselaar T.G. \& Gibson G. Antigenic relationship between chikungunya virus strains and o'nyong nyong virus using monoclonal antibodies. Res Virol, 1995; 146: 69-73

Bordi L., Carletti F., Castilletti C. et al. Presence of the A226V mutation in autochthonous and imported Italian chikungunya virus strains. Clin Infect Dis, 2008; 47: 428-429

Bordi L., Meschi S., Selleri M., et al. Chikungunya virus isolates with/without A226V mutation show different sensitivity to IFN-a, but similar replication kinetics in non human primate cells. New Microbiol, 2011; 34: 87-91

Brighton S.W., Prozesky O.W. \& de la Harpe A.L. Chikungunya virus infection. A retrospective study of 107 cases. S Afr Med J, 1983; 63: 313-315

Brighton S.W. Chloroquine phosphate treatment of chronic Chikungunya arthritis: an open pilot study. S Afr Med J, 1984; 66: 217-218

Briolant S., Garin D., Scaramozzino N. et al. In vitro inhibition of Chikungunya and Semliki Forest viruses replication by antiviral compounds: synergistic effect of interferonalpha and ribavirin combination. Antiviral Res, 2004; 61: 111-117

Carletti F., Bordi L., Chiappini R., et al. Rapid detection and quantification of Chikungunya virus by a one-step reverse transcription polymerase chain reaction real-time assay. Am J Trop Med Hyg, 2007; 77: 521-524. 
Centers for Disease Control and Prevention (CDC).Chikungunya fever diagnosed among international travelers--United States, 2005-2006. MMWR Morb Mortal Wkly Rep., 2006; 55: 1040-2.

Centers for Disease Control and Prevention (CDC). Update: chikungunya fever diagnosed among international travelers--United States, 2006. MMWR Morb Mortal Wkly Rep., 2007; 56:276-7.

Charrel R.N., de Lamballerie X. \& Raoult D. Chikungunya outbreaks--the globalization of vector-borne diseases. N Engl J Med, 2007; 356: 769-771

Charrel R. \& de Lamballerie X. Chikungunya in north-eastern Italy: a consequence of seasonal synchronicity. Euro Surveill, 2008; 13: pii: 8003

Chatterjee S.N., Chakravarti S.K., Mitra A.C. et al. Virological investigation of cases with neurological complications during the outbreak of haemorrhagic fever in Calcutta. J Indian Med Assoc, 1965; 45: 314-316

Chatterjee S.N. \& Sarkar J.K. Electron microscopic studies of suckling mouse brain cells infected with Chikungunya virus. Indian. J Exp Biol, 1965; 3: 227-234

Cordel H., Quatresous I., Paquet C. et al. Imported cases of chikungunya in metropolitan France, April 2005 - February 2006. Euro Surveill, 2006; 11: E060420.3

Couderc T., Chrétien F., Schilte C. et al. A mouse model for Chikungunya: young age and inefficient Type-I interferon signalling are risk factors for severe disease. Plos Pathogen, 2008; 4: e29

Couderc T., Khandoudi N., Grandadam M. et al. Prophylaxis and therapy for Chikungunya virus infection. J Infect Dis, 2009; 200: 516-523

Cui F., Raymond M. \& Qiao C.L. . Insecticide resistance in vector mosquitoes in China. Pest Manag Sci, 2006; 62: 1013-1022

D'Ortenzio E., Grandadam M., Balleydier E. et al. Sporadic cases of chikungunya, Reunion Island, August 2009. Euro Surveill, 2009; 14: pii: 19324

D'Ortenzio E., Grandadam M., Balleydier E. et al. A226V strains of Chikungunya virus, Réunion Island, 2010. Emerg Infect Dis, 2011; 17: 309-311

de Lambellerie X., Leroy E., Charrel R.N. et al. Chikungunya virus adapts to tiger mosquito via evolutionary convergence: a sign of things to come? Virol J, 2008; 5: 33

de Lamballerie X., Boisson V., Reynier J.C. et al. On chikungunya acute infection and chloroquine treatment. Vector Borne Zoonotic Dis, 2008; 8: 837-839

Depoortere E., \& Coulombier D. Chikungunya risk assessment for Europe: recommendations for action. Euro Surveill, 2006; 11: E060511.2

Dessain S.K., Adekar S.P., \& Berry J.D. Exploring the native human antibody repertoire to create antiviral therapeutics. Curr Top Microbiol Immunol, 2008; 317: 155-183

Eckels K.H. Harrison V.R. \& Hetrick .FM. Chikungunya virus vaccine prepared by Tweenether extraction. Appl Microbiol, 1970; 19: 321-325

Edelman R., Tacket C.O., Wasserman S.S. et al. Phase II safety and immunogenicity study of live Chikungunya virus vaccine TSI-GSD-218. Am J Trop Med Hyg, 2000; 62: 681685

Enserink M. Infectious diseases. Massive outbreak draws fresh attention to little-known virus. Science, 2006; 311: 1085 
Fourie E.D. \& Morrison J.G. Rheumatoid arthritic syndrome after chikungunya fever. S Afr Med J, 1979; 56: 130-132

Fusco F.M., Puro V., Di Caro A. et al. Cases of Chikungunya fever in Italy in travellers returning from the Indian Ocean and risk of introduction of the disease to Italy. Infez Med, 2006; 14: 238-245

Fusco F.M. et al. (2010). Chikungunya fever, a re-emerging disease, In: Tropical and Emerging Infectious Diseases, Maltezou Helen C. and Achilleas Gikas, 93-110, ISBN: 978-81308-0389-0

Gubler D.J., Reiter P., Ebi K.L. et al. Climate variability and change in the United States: potential impacts on vector- and rodent-borne diseases. Environ Health Perspect, 2001; 109 Suppl 2: 223-233

Harrison V.R., Binn L.N. \& Randall R. Comparative immunogenicities of chikungunya vaccines prepared in avian and mammalian tissues. Am J Trop Med Hyg, 1967; 16: 786-791

Harrison V.R, Eckels K.H., Bartelloni P.J. et al. Production and evaluation of a formalinkilled Chikungunya vaccine. J Immunol, 1971; 107: 643-647

Hearn H.J. Jr. \& Rainey C.T. Cross-protection in animals infected with Group A arboviruses. J Immunol, 1963; 90: 720-724

Her Z., Malleret B., Chan M. et al. Active Infection of Human Blood Monocytes by Chikungunya Virus Triggers an Innate Immune Response. J Immunol, 2010; 184: 5903-5913

Higgs S. The 2005-2006 chikungunya epidemic in the Indian Ocean. Vector Borne Zoonotic Dis 2006; 6: 115-116

Hochedez P., Jaureguiberry S., Debruyne M. et al. Chikungunya infection in travelers. Emerg Infect Dis, 2006; 12: 1565-1567

Hochedez P., Hausfater P., Jaureguiberry S. et al. Cases of chikungunya fever imported from the islands of the South West Indian Ocean to Paris, France: 80 cases in France. Euro Surveill, 2007; 12: [Epub ahead of print]

Inoue S., Morita K., Matias R.R. et al. Distribution of three arbovirus antibodies among monkeys (Macaca fascicularis) in the Philippines. J Med Primatol, 2003; 32: 89-94

Jacups S.P., Whelan P.I. \& Currie B.J. Ross River virus and Barmah Forest virus infections: a review of history, ecology, and predictive models, with implications for tropical northern Australia. Vector Borne Zoonotic Dis, 2008; 8: 283-297

Jain M., Rai S. \& Chakravarti A. Chikungunya: a review. Trop Doc, 2008; 38: 70-72

Jeandel P., Josse R. \& Durand J.P. Exotic viral arthritis: role of alphavirus. Med Trop, 2004; 64: 81-88 (in French)

Josseran L., Paquet C., Zehgnoun A. et al. Chikungunya disease outbreak, Reunion Island. Emerg Infect Dis, 2006; 12: 1994-1995

Jupp P.G., McIntosh B.M., Dos Santos I. et al. Laboratory vector studies on six mosquito and one tick species with chikungunya virus. Trans R Soc Trop Med Hyg, 1981; 75: 1519

Jupp P.G. \& McIntosh B.M. Aedes furcifer and other mosquitoes as vectors of chikungunya virus at Mica, northeastern Transvaal, South Africa. J Am Mosq Control Assoc, 1990; 6: 415-420 
Kam Y.W., K.S. Ong E., Laurent R et al. Immuno-biology of Chikungunya and implications for disease intervention. Microbes Infect, 2009; 11: 1186-1196

Kennedy A.C., Fleming J. \& Solomon L. Chikungunya viral arthropathy: a clinical description. J Rheumatol, 1980; 7: 231-236

Kenney J.L., Volk S.M., Pandya J., et al. Stability of RNA virus attenuation approaches. Vaccine, 2011; 29: 2230-4.

Khan A.H., Morita K., Parquet Md Mdel C. et al. Complete nucleotide sequence of Chikungunya virus and evidence for an internal polyadenylation site. Gen Virol, 2002; 83, 3075-3084

Knudsen A.B. Global distribution and continuing spread of Aedes albopictus. Parassitologia, 1995; 37: 91-97

Labadie K., Larcher T., Joubert C. et al. Chikungunya disease in nonhuman primates involves long-term viral persistence in macrophages. J Clin Invest, 2010; 120: 894906

Lakshmi V., Neeraja M., Subbalaxmi M.V. et al. Clinical features and molecular diagnosis of Chikungunya fever from South India. Clin Infect Dis, 2008; 46: 1436-1442

Lam S.K., Chua K.B., Hooi P.S. et al. Chikungunya infection-an emerging disease in Malaysia. Southeast Asian J Trop Med Public Health , 2001; 32: 447-451

Lebrun G., Chadda K., Reboux A.H. et al. Guillain-Barré syndrome after chikungunya infection. Emerg Infect Dis, 2009; 15: 495-496

Lemant J., Boisson V., Winer A. et al. Serious acute Chikungunya virus infection requiring intensive care during the Reunion Island outbreak in 2005-2006. Crit Care Med, 2008; 36: 2536-2541

Leo Y.S., Chow A.L.P., Tan L.K. et al. Chikungunya Outbreak, Singapore, 2008. Emerg Infect Dis, 2009 15: 836-837

Levitt N.H., Ramsburg H.H., Hasty S.E. et al. Development of an attenuated strain of chikungunya virus for use in vaccine production. Vaccine, 1986; 4: 157-162

Lidbury B.A., Rulli N.E., Suhrbier A. et al. Macrophage-derived proinflammatory factors contribute to the development of arthritis and myositis after infection with an arthrogenic alphavirus. J Infect Dis, 2008; 197: 1585-1593

Litzba N., Schuffenecker I., Zeller H. et al.Evaluation of the first commercial chikungunya virus indirect immunofluorescence test. J Virol Methods, 2008; 149: 175-179

Mahalingam S., Meanger J., Foster P.S. et al. The viral manipulation of the host cellular and immune environments to enhance propagation and survival: a focus on RNA viruses. J Leukoc Biol, 2002; 72: 429-439

Mahendradas P., Ranganna S.K., Shetty R. et al. Ocular manifestations associated with chikungunya. Ophthalmol, 2008; 115: 287-291

Mallilankaraman K., Shedlock D.J., Bao H. et al. A DNA vaccine against chikungunya virus is protective in mice and induces neutralizing antibodies in mice and nonhuman primates. PLoS Negl Trop Dis, 2011; 5: e928

Mavalankar D., Shastri P. \& Raman P. Chikungunya epidemic in India: a major publichealth disaster. Lancet Infect Dis, 2007; 7: 306-307

Mavalankar D., Shastri P., Bandyopadhyay T. et al. Increased mortality rate associated with Chikungunya epidemic, Ahmedabad, India. Emerg Infect Dis, 2008; 14: 412-415 
Muthumani K., Lankaraman K.M., Laddy D.J. et al. Immunogenicity of novel consensusbased DNA vaccines against Chikungunya virus. Vaccine, 2008; 26: 5128-5134

Ng L.F.P., Chow A., Sun Y.J. et al. IL-1b, IL-6,and RANTES as biomarkers of Chikungunya severity. PLoS One, 2009; 4: e4261

Ozden S., Huerre M., Riviere J.P. et al. Human muscle satellite cells as targets of Chikungunya virus infection. PLoS One, 2007; 2: e527

Panning M., Grywna K., van Esbroeck, M. et al. Chikungunya fever in travelers returning to Europe from the Indian Ocean region, 2006. Emerg Infect Dis, 2008; 14: 416-422

Parola P., de Lamballerie X., Jourdan J. et al. Novel Chikungunya virus variant in travellers returning from Indian Ocean islands. Emerg Infect Dis, 2006; 12: 1493-1499

Pedersen C.E, Robinson D.M., Cole F.E. Isolation of the vaccine strain of Venezuelan equine encephalomyelitis virus from mosquitoes in Louisiana. Am J Epidemiol, 1972; 95:490-496

Pfeffer M., Hanus I., Löscher T. et al. Chikungunya fever in two German tourists returning from the Maldives, September, 2009. Euro Surveill, 2010; 15: pii: 19531

Pialoux G., Gaüzère B.A., Jauréguiberry S. et al. Chikungunya, an epidemic arbovirosis. Lancet Infect Dis, 2007; 7: 319-327

Pile J.C., Henchal E.A., Christopher G.W. et al. Chikungunya in a North American traveler. J Travel Med, 1999; 6: 137-139

Pistone T., Ezzedine K., Schuffenecker I., et al. An imported case of Chikungunya fever from Madagascar: use of the sentinel traveller for detecting emerging arboviral infections in tropical and European countries. Travel Med Infect Dis, 2009;7: 52-4.

Plante K., Wang E., Partidos C.D., et al. Novel Chikungunya Vaccine Candidate with an IRES-Based Attenuation and Host Range Alteration Mechanism. PLoS Pathog, 2011; 7 : e1002142.

Poletti P., Messeri G., Ajelli M. et al. Transmission potential of chikungunya virus and control measures: the case of Italy. PLoS One, 2011; 6: e18860

Powers A.M., Brault A.C., Tesh R.B. et al. Re-emergence of chikungunya and o'nyong-nyong viruses: evidence for distinct geographical lineages and distant evolutionary relationships. J Gen Virol, 2000; 81: 471-479

Precious S.W. Webb H.E. \& Bowen E.T.W. Isolation and persistence of Chikungunya virus in cultures of mouse brain cells. J Gen Virol, 1974; 23: 271-279

Quatresous I. E-alert 27 January: chikungunya outbreak in Reunion, a French overseas department. Euro Surveill 2006; 11: E060202.1

Ravi V. Re-emergence of chikungunya virus in India. Indian J Med Microbiol, 2006; 24: 83-84

Ravichandran R. \& Manian M. Ribavirin therapy for Chikungunya arthritis. J Infect Dev Ctries, 2008; 2: 140-142.

Receveur M., Ezzedine K., Pistone T. et al. Chikungunya infection in a French traveller returning from the Maldives, October, 2009. Euro Surveill, 2010; 15: 19494

Reiter P., Fontenille D. \& Paupy C. Aedes albopictus as an epidemic vector of chikungunya virus: another emerging problem? Lancet Infect Dis, 2006; 6: 463-464

Rezza G., Nicoletti L., Angelini R. et al. CHIKV study group. Infection with Chikungunya virus in Italy: an outbreak in a temperate region. Lancet, 2007; 370: 1840-1846 
Robinson M.C. An epidemic of virus disease in Southern Province, Tanganyika Territory, in 1952-1953. I. Clinical features. Trans Royal Society Trop Med Hyg, 1955; 49:28-32

Sam I.C. \& AbuBakar S. Chikungunya virus infection. Med J Malaysia 2006; 61: 264-269

Saxena S., Singh M., Mishra N et al. Resurgence of chikungunya virus in India: an emerging threat. Euro Surveill, 2006; 11: E060810.2

Schilte C., Couderc T., Chretien F. et al. Type I IFN controls chikungunya virus via its action on non-hematopoietic cells. J Exp Med, 2010; 207: 429-442

Schuffenecker I., Iteman I., Michault A. et al. Genome microevolution of Chikungunya viruses causing the Indian Ocean outbreak. Plos Medicine, 2006; 3: 1058-1070

Sourisseau M., Schilte C., Casartelli N. et al. Characterization of reemerging chikungunya virus. PLoS Pathogen, 2007; 3: e89

Strauss J.H., Strauss E.M. The alphaviruses: gene expression, replication and evolution. Microbiol Rev, 1994; 58: 491-562

Taubitz W., Cramer J.P., Kapaun A. et al. Chikungunya fever in travelers: clinical presentation and course. Clin Infect Dis, 2007; 45: e1-4

Tsetsarkin K.A., Vanlandingham D.L., McGee C.E. et al. A Single Mutation in Chikungunya Virus Affects Vector Specificity and Epidemic Potential. PLoS Pathog, 2007; 3: 18951906

Vazeille M., Moutailler S., Coudrier D. et al. Two Chikungunya Isolates from the Outbreak of La Reunion (Indian Ocean) Exhibit Different Patterns of Infection in the Mosquito, Aedes albopictus. PLoS One, 2007; 2: 1-9

Wang E., Volkova E., Adams A.P. et al. Chimeric alphavirus vaccine candidates for chikungunya. Vaccine, 2008; 26: 5030-5039

Wang D., Suhrbier A., Penn-Nicholson A. et al. A complex adenovirus vaccine against chikungunya virus provides complete protection against viraemia and arthritis. Vaccine, 2011; 29: 2803-2809

Wang E., Kim D.Y., Weaver S.C., et al. Chimeric Chikungunya Viruses are Nonpathogenic in Highly Sensitive Mouse Models, but Efficiently Induce a Protective Immune Response. J Virol, 2011 Jun 22. [Epub ahead of print]

Warter L., Lee C.Y., Thiagarajan R. et al. Chikungunya virus envelope-specific human monoclonal antibodies with broad neutralization potency. J Immunol, 2011; 186: 3258-3264

Wauquier N., Becquart P., Nkoghe D. et al. The acute phase of chikungunya virus infection in humans is associated with strong innate immunity and T CD8 cell activation. J Infect Dis, 2011; 204: 115-123

White A., Berman S. \& Lowenthal J.P. Comparative immunogenicities of Chikungunya vaccines propagated in monkey kidney monolayers and chick embryo suspension cultures. Appl Microbiol, 1972; 23: 951-952

Wielanek A.C., Monredon J.D., Amrani M.E. et al. Guillain-Barré syndrome complicating a Chikungunya virus infection. Neurology, 2007; 69: 2105-2107

Wolfe N.D., Kilbourn A.M., Karesh W.B. et al. Sylvatic transmission of arboviruses among Bornean orangutans. Am J Trop Med Hyg, 2001; 64: 310-316

Yergolkar P., Tandale B., Arankalle V. et al. Chikungunya outbreaks caused by African genotype, India. Emerg Infect Dis, 2006; 12: 1580-1583 
Zeller H.G. Dengue, arbovirus and migrations in the Indian Ocean. Bull Soc Pathol Exot, 1998; 91: 56-60

Ziegler S.A., Lu L.,. da Rosa A.P. et al. An animal model for studying the pathogenesis of Chikungunya virus infection. Am J Trop Med Hyg, 2008; 79: 133-139 


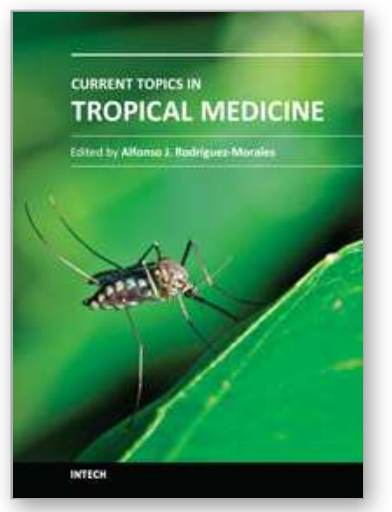

\author{
Current Topics in Tropical Medicine \\ Edited by Dr. Alfonso Rodriguez-Morales
}

ISBN 978-953-51-0274-8

Hard cover, 564 pages

Publisher InTech

Published online 16, March, 2012

Published in print edition March, 2012

Tropical Medicine has emerged and remained as an important discipline for the study of diseases endemic in the tropic, particularly those of infectious etiology. Emergence and reemergence of many tropical pathologies have recently aroused the interest of many fields of the study of tropical medicine, even including new infectious agents. Then evidence-based information in the field and regular updates are necessary. Current Topics in Tropical Medicine presents an updated information on multiple diseases and conditions of interest in the field. It Includes pathologies caused by bacteria, viruses and parasites, protozoans and helminths, as well as tropical non-infectious conditions. Many of them are considering not only epidemiological aspects, but also diagnostic, therapeutical, preventive, social, genetic, bioinformatic and molecular ones. With participation of authors from various countries, many from proper endemic areas, this book has a wide geographical perspective. Finally, all of these characteristics, make an excellent update on many aspects of tropical medicine in the world.

\title{
How to reference
}

In order to correctly reference this scholarly work, feel free to copy and paste the following:

Bordi Licia, Meschi Silvia, Selleri Marina, Lalle Eleonora, Castilletti Concetta, Carletti Fabrizio, Di Caro Antonino and Capobianchi Maria Rosaria (2012). The Re-Emergence of an Old Disease: Chikungunya Fever, Current Topics in Tropical Medicine, Dr. Alfonso Rodriguez-Morales (Ed.), ISBN: 978-953-51-0274-8, InTech, Available from: http://www.intechopen.com/books/current-topics-in-tropical-medicine/the-re-emergence-of-an-olddisease-chikungunya-fever

\section{INTECH}

open science | open minds

\section{InTech Europe}

University Campus STeP Ri

Slavka Krautzeka 83/A

51000 Rijeka, Croatia

Phone: +385 (51) 770447

Fax: +385 (51) 686166

www.intechopen.com

\section{InTech China}

Unit 405, Office Block, Hotel Equatorial Shanghai

No.65, Yan An Road (West), Shanghai, 200040, China 中国上海市延安西路65号上海国际贵都大饭店办公楼405单元

Phone: +86-21-62489820

Fax: +86-21-62489821 
(C) 2012 The Author(s). Licensee IntechOpen. This is an open access article distributed under the terms of the Creative Commons Attribution 3.0 License, which permits unrestricted use, distribution, and reproduction in any medium, provided the original work is properly cited. 